\section{Teach the young, teach everyone}

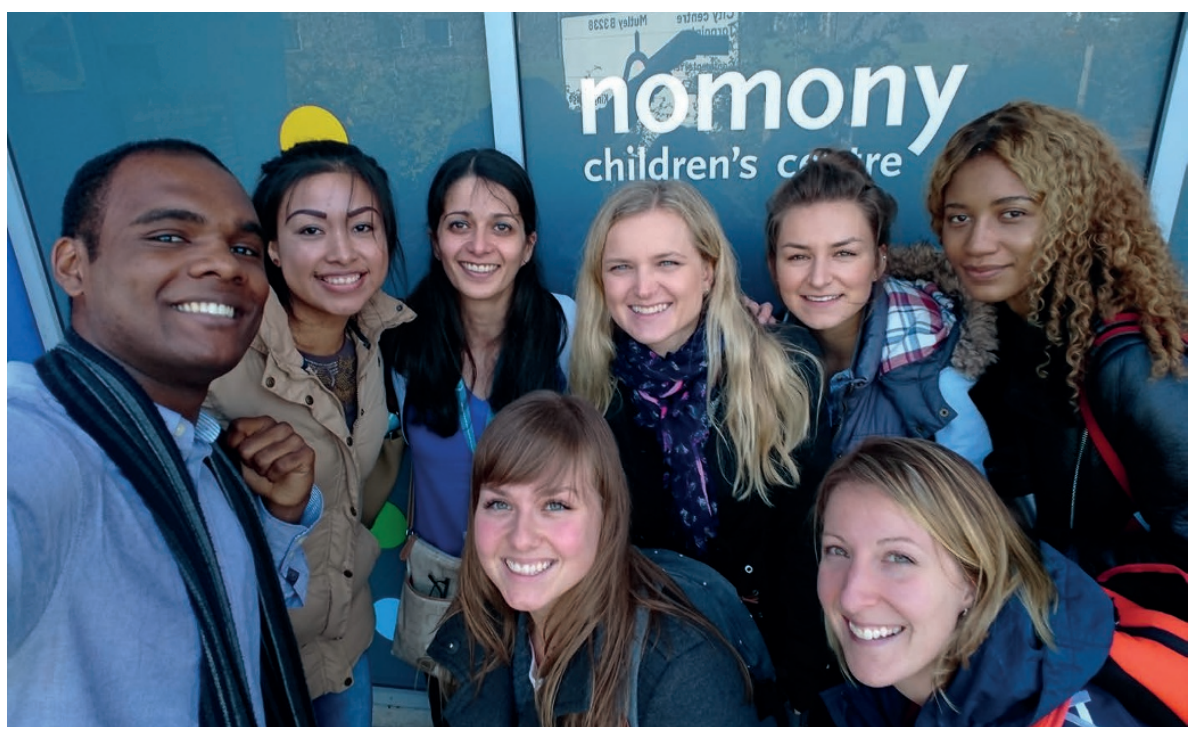

'Inter-Professional Engagement' is a module at Peninsula Dental School of Plymouth

University that Year 2 Dental Therapy and Hygiene students undertake as part of training.

The design of this module is to give students the opportunity to work alongside a communitybased organisation to deliver an intervention project which supports positive lifestyle choices and health improvement for a target population group. This promotes skill development and understanding outside of the clinical environment. The charity Well Connected acts as the link between the University and the community organisations.

A target population of children aged $0-5$ years was allocated to a group in the academic year 2017-18 (pictured). This was with the charity Barnardo's who run Nomony Children's Centre, which has been set up in Plymouth to transform the lives of disadvantaged children and their families. The aims of the project were to improve knowledge and raise awareness of the importance of good oral health in young children and their families who attend the Children's Centre. This was done by:

- Delivering two key oral health messages: spit, don't rinse and brush twice a day for two minutes with a fluoride toothpaste

- Promoting good oral hygiene by providing dental starter kits

- Promoting good dietary habits by demonstrating healthy 'low sugar' snacks and encouraging reducing sugar to meal times

- Using signposting methods to inform families of places to access dental care

- Reinforcing key oral health messages by performing a familiar sounding nursery rhyme.

Due to the target population being aged $0-5$ years, the group decided the project had to be interactive and entertaining. With task delegation, it was one individual's (GS) responsibility to write the lyrics of the song. Repetitive verses and a familiar sounding nursery rhyme were used to consolidate learning and emphasise key oral health messages based on 'Delivering better oral health' guidelines. Hence, 'The Wheels on the Bus' tune was used!

This song (pictured right) has been left with Nomony Children's Centre where they continue to use it regularly at the end of their group sessions. Furthermore, it is currently being distributed by Well Connected charity to early year partners in Plymouth. The song can be used free of charge.

By Georgia Snape

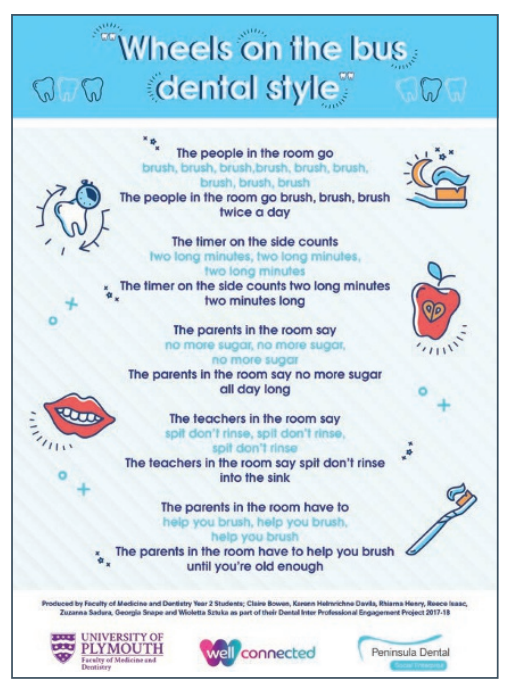

\section{Call out to dentists and DCPs!}

Cariology researchers from Dundee and Berlin are looking for dentists and DCPs to take part in a study to find out which study outcomes are most relevant to day-to-day clinical practice.

The project will use a series of online surveys to develop consensus around what outcomes should be routinely measured in trials investigating treatment of carious lesions. By seeking the views of patients and clinicians, the aim is to ensure that clinical trials provide relevant answers to questions that clinicians and patients have when making treatment choices.

This series of online questionnaires and feedback to participants (eDelphi) will result in a core list of outcomes. The routine use of this evidence-based core outcome set in future clinical trials will help with evidence synthesis and comparison of results between studies.

Please consider taking part and using your experience to shape the future priorities of caries research. Three survey rounds are expected, each requiring a time commitment of about 20 minutes. The first round of the survey will be open from 4 July 2018 and run for 4-5 weeks.

If you are interested in taking part and would like more information, please contact the project lead, Colin Levey at c.levey@dundee.ac.uk. 